\title{
FURTHER INTERPOLATION INEQUALITIES RELATED TO ARITHMETIC-GEOMETRIC MEAN, CAUCHY-SCHWARZ AND HÖLDER INEQUALITIES FOR UNITARILY INVARIANT NORMS
}

\author{
MOHAMmAD AL-KHLYLEH AND FADI ALRIMAWI
}

Abstract. An inequality for matrices that interpolates between the Cauchy-Schwarz and the arithmetic-geometric mean inequalities for unitarily invariant norms has been obtained by Audenaert. Alakhrass obtained a related result to Audenaert's inequality using a log-convex function $g$ defined on $[0,1]$. Very recently, Zou obtained an inequality for matrices that unifies Hölder's inequality and the arithmetic-geometric mean inequality for unitarily invariant norms. A generalized version of Zou's inequality for unitarily invariant norms is given, and an alternative proof of Audenaert's inequality using a refined version of Alakhrass's function is presented.

Mathematics subject classification (2010): 15A18, 15A60, 47A30.

Keywords and phrases: Singular value, unitarily invariant norm, inequality, interpolation.

\section{REFERENCES}

[1] M. Alakhrass, A note on Audenaert interpolation inequality, Linear and Multilinear Algebra, 66, 9 (2018), 1909-1916.

[2] M. Al-Khlyleh And F. KitTANeh, Interpolating inequalities related to a recent result of Audenaert, Linear and Multilinear Algebra, 65, 5 (2017), 922-929.

[3] T. Ando, Matrix Young inequality, Oper. Theory Adv. Appl., 75, (1995), 33-38.

[4] K.M.R. AudenAert, Interpolating between the arithmetic-geometric mean and Cauchy-Schwarz matrix norm inequalities, Oper. Matrices, 9, (2015), 475-479.

[5] R. Bhatia, Matrix Analysis, Springer-Verlag, New York, 1997.

[6] R. BHATIA AND C. DAVIS, More matrix forms of the arithmetic-geometric mean inequality, SIAM J. Matrix Anal. Appl., 14, (1993), 132-136.

[7] R. Bhatia AND C. DAvis, A Cauchy-Schwarz inequality for operators with applications, Linear Algebra Appl., 223, (1995), 119-129.

[8] R. Bhatia And F. Kittaneh, On the singular values of a product of operators, SIAM J. Matrix Anal. Appl., 11, (1990), 272-277.

[9] R.A. Horn AND R. MATHIAS, Cauchy-Schwarz inequalities associated with positive semidefinite matrices, Linear Algebra Appl., 142, (1990), 63-82.

[10] J.C. BOURIN AND E. Y. LEE, Matrix inequalities from a two variables functional, International Journal of Mathematics, 27, 09 (2016), p.1650071.

[11] R.A. HORN AND X. ZHAN, Inequalities for CS seminorms and Lieb functions, Linear algebra appl., 291, 1-3 (1999), 103-113.

[12] F. KitTANEH, A note on the arithmetic-geometric mean inequality for matrices, Linear Algebra Appl., 171, (1992), 1-8.

[13] H. Kos AKI, Arithmetic-geometric mean and related inequalities for operators, Journal of Functional Analysis, 156, 2 (1998), 429-451.

[14] X. Zhan, Matrix Inequalities, Springer-Verlag, Berlin, 2002.

[15] L. ZOU, Unification of the arithmetic-geometric mean and Hölder inequalities for unitarily invariant norms, Linear Algebra Appl., 562, (2019), 154-162. 